\title{
Cervical Symmetric Dumbbell Ganglioneuromas Causing Severe Paresis
}

\author{
Akira Hioki $^{1}$, Kei Miyamoto ${ }^{2}$, Yoshinobu Hirose ${ }^{3}$, Yusuke Kito ${ }^{3}$, Kazunari Fushimi ${ }^{1}$, Katsuji Shimizu ${ }^{1}$ \\ ${ }^{1}$ Department of Orthopaedic Surgery, Gifu University Graduate School of Medicine, Gifu, Japan \\ ${ }^{2}$ Department of Reconstructive Surgery for Spine, Bone, and Joint, Gifu University Graduate School of Medicine, Gifu, Japan \\ ${ }^{3}$ Department of Pathology, Gifu University Graduate School of Medicine, Gifu, Japan
}

We report an extremely rare case with bilateral and symmetric dumbbell ganglioneuromas of the cervical spine in an elderly patient. A 72-year-old man came by ambulance to our hospital due to progressive incomplete paraplegia. Magnetic resonance imaging demonstrated bilateral symmetric dumbbell tumors at the $\mathrm{C} 1 / 2$ level. We performed total resection of the intracanalar tumor, aiming at complete decompression of the spinal cord, and partial and subtotal resection of foraminal outside portions. Histopathological examination of the surgical specimen indicated the tumor cells to be spindle cells with the presence of ganglion cells and no cellular pleomorphism, suggesting a diagnosis of ganglioneuroma. Although the surgery was not curative, the postoperative course was uneventful and provided a satisfactory outcome. This is the fourth known case of cervical ganglioneuromas of the bilateral symmetric dumbbell type.

Keywords: Ganglioneuroma; Symmetric; Dumbbell tumor; Cervical spine

\section{Introduction}

Ganglioneuromas are rare, slow-growing benign tumors that usually develop from the sympathetic nervous system, of which $60 \%$ occur in children and young adults [1-3]. They are most commonly found in the posterior mediastinum and abdomen, in the lumbar and pelvic retroperitoneal space. Occasionally, they grow into the spinal canal extradurally and become dumbbell shaped. Cervical ganglioneuromas are also rare, and to the best of our knowledge, only ten cases have been reported [110]. In cervical ganglioneuromas, the bilateral symmetric dumbbell type is even more rare, comprising only three reported cases [1-3]. The purpose of this report was to present an additional case with bilateral and symmetric dumbbell ganglioneuromas of the cervical spine in an elderly patient.

\section{Case Report}

A 72-year-old man came by ambulance to our hospital due to progressive incomplete paraplegia. One month previously, he had felt left upper extremity numbness. He could not stand alone or walk because of sudden onset lightheadedness and reduced muscular strength in both legs. Manual muscle test of the right side arm and leg (below $\mathrm{C} 2$ ) was graded as 4- and that of the left side arm and leg (below C2) was graded as 4 . He showed bladder dysfunction. He had incomplete sensory loss below C3. Tonus was increased in both legs and deep tendon reflex-

Received Jul 12, 2012; Revised Sep 18, 2012; Accepted Oct 22, 2012

Corresponding author: Akira Hioki

Department of Orthopaedic Surgery, Gifu University Graduate School of Medicine,

1-1 Yanagido, Gifu 501-1194, Japan

Tel: +81-58-230-6333, Fax: +81-58-230-6334, E-mail: akira-hi@amber.plala.or.jp 
es were hyperactive. In addition, he had been diagnosed with gastric cancer and had undergone total gastrectomy and splenectomy, resulting in peritoneal dissemination.

Magnetic resonance imaging (MRI) demonstrated bilateral asymmetric masses at the $\mathrm{C} 1 / 2$ level. T1-weighted images exhibited hypointensity. T2-weighted images revealed heterogeneous hyperintensity (Fig. 1A-C). Contrast-enhanced T1-weighted images showed enhancement in the tumor parenchyma (Fig. 1D, E). Computed tomography myelogram showed spinal cord compression bilaterally at $\mathrm{C} 1 / 2$. Because the progression of the paralysis was rapid and the patient hoped for a palliative surgical procedure, we performed emergency operation to remove the intracanalar tumor and decompress the spinal cord.

\section{Operative findings}

After the posterior arch at $\mathrm{C} 1$ and cranial portion of the $\mathrm{C} 2$ lamina was removed and the dura was exposed (Fig. $2 \mathrm{~A}$ ), extradural bilateral tumor masses at $\mathrm{C} 1 / 2$ were identifiable (Fig. 2B, C). Tumors surface were smooth with capsules. Firstly the extradural tumor was partially resected and the debulking effect obtained. Bilateral C2 dorsal roots were swollen, with cancerization and connected intradural portions. After durotomy, bilateral symmetric tumors originating from bilateral C2 dorsal root were identified (Fig. 2D, E). These were partially adherent to the dura. The intradural portions of the tumors were totally resected piece by piece. Consequently, C2 bilateral dorsal roots were sacrificed. Subtotal resection for the bilateral extradural portions was then carried out. It was confirmed that that intracanalar portions were totally resected and the emergency operation was thus terminated, because radical resection for the extracanalar portions, which would have required dissection of the tumor from vertebral arteries, was not deemed appropriate given the patient's relatively poor general condition. Finally, we performed duraplasty with artificial dura mater to extend the intradural space.

Histopathological examination of the surgical specimen indicated the tumor cells to be spindle cells with the presence of ganglion cells and no cellular pleomorphism. Ganglion cells were dissipated throughout the tumor (Fig. 3). The tumors were thus diagnosed as ganglioneuromas. After the operation, the patient's paraplegia improved
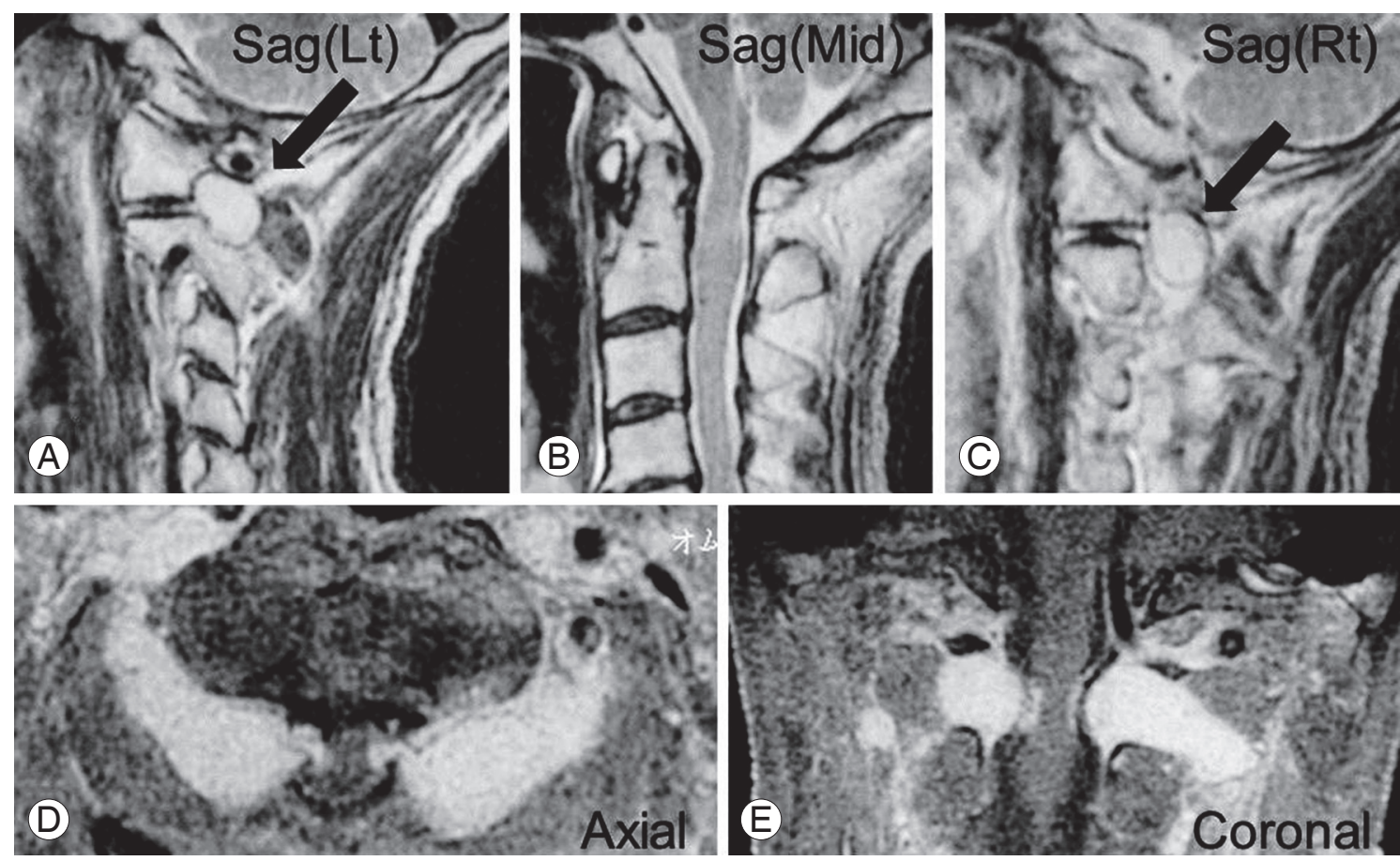

Fig. 1. Preoperative magnetic resonance images. T2-weighted sagittal images. (A) Left side para-sagittal section, (B) mid-sagittal section, (C) right side para-sagittal section, revealed a heterogeneous hyperintensity (black arrow on [A] and [C]). Contrastenhanced T1-weighted images. (D) Axial section at C1/2, (E) coronal section of spinal cord at C1/2, showed enhancement in the tumor parenchyma. 
gradually and he became able to walk. MRI after operation showed that bilateral foraminal portions were left intact and the intracanal tumor was completely resected, with sufficient spinal cord decompression (Fig. 4). He was followed up with MRI at three month intervals. Two years postoperatively, there was no recurrence intraca- nally and no increase in the foraminal portions. Taking into account his poor general condition, including cancerous ascites due to the peritoneal dissemination of past gastric cancer, we are not planning to perform an additional operation as long as his improvement in paresis is maintained.

\section{Gross appearance after C1-2 laminectomy}

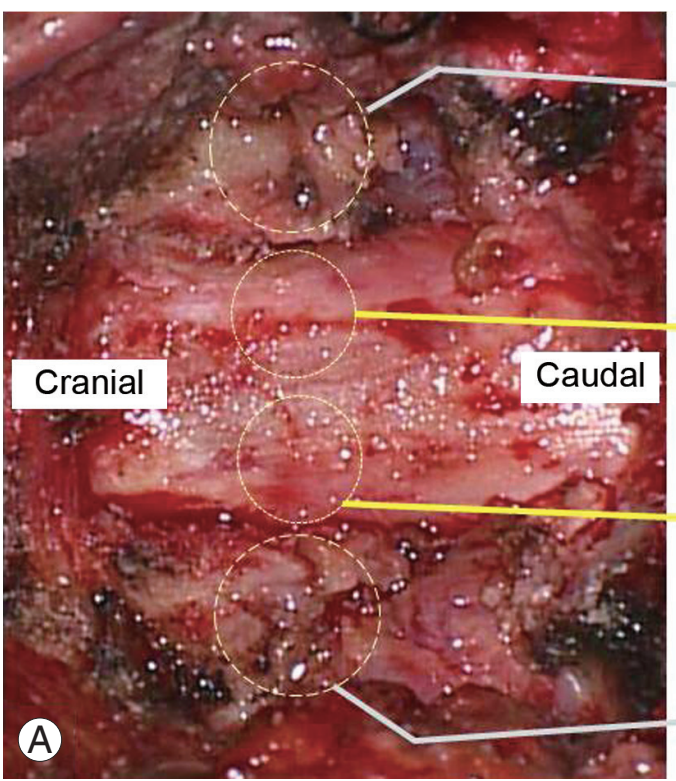

Extradural portion
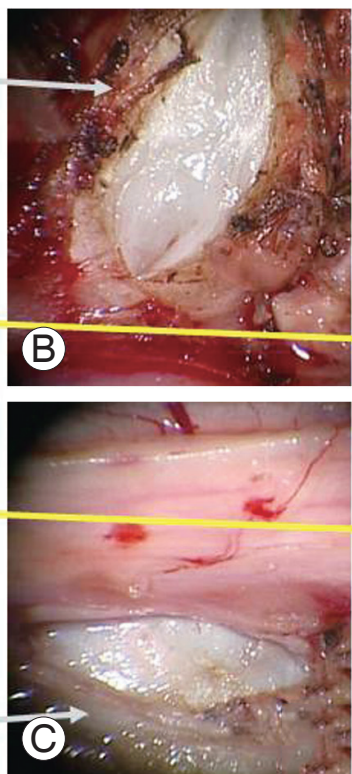

Intradural

portion
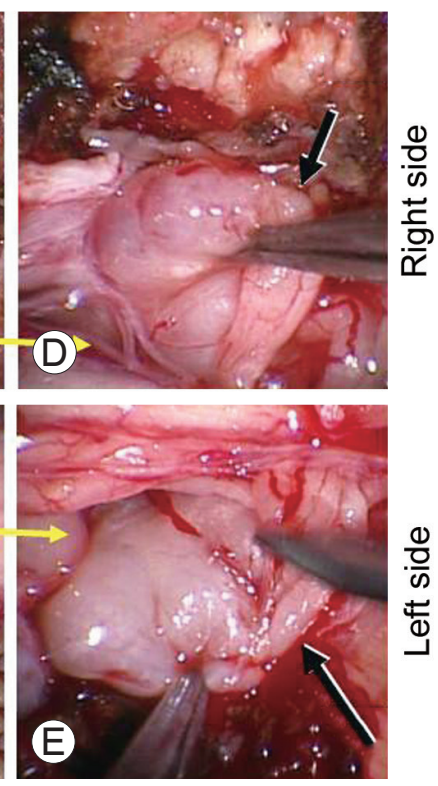

Fig. 2. Intraoperative findings. After the posterior arch at C1 and cranial portion of the C2 lamina were removed and the dura was exposed (A), extradural bilateral tumor masses at C1/2 were identifiable (extradural portions of the tumor at right side [B] and left side [C]). After durotomy, bilateral symmetric tumors originating from the bilateral C2 dorsal root were identified (intradural portions of the tumor at right side [D, black arrow] and left side [E, black arrow]).

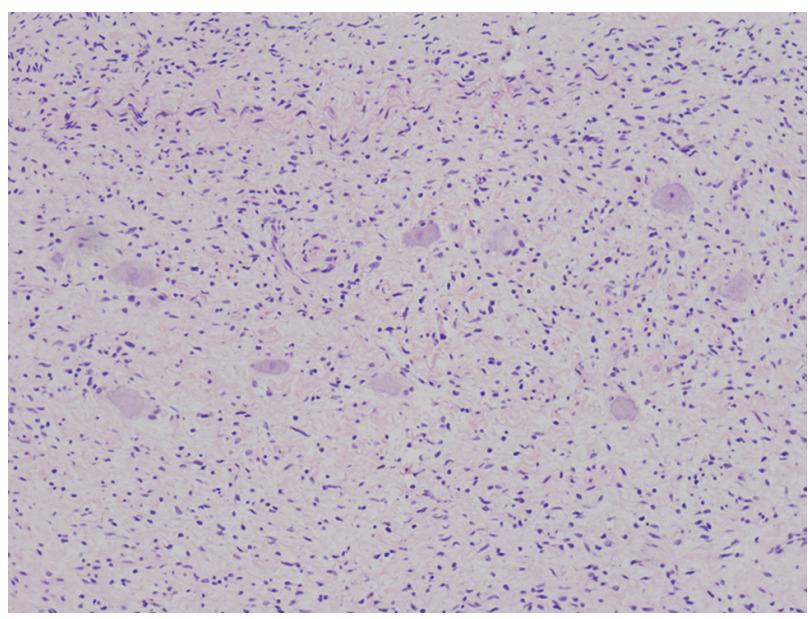

Fig. 3. Histopathological examination. The tumor cells were found to be spindle cells with no cellular pleomorphism and presence of ganglion cells. Ganglion cells were dissipating throughout the tumor $(H \& E$, $\mathrm{x} 100)$.

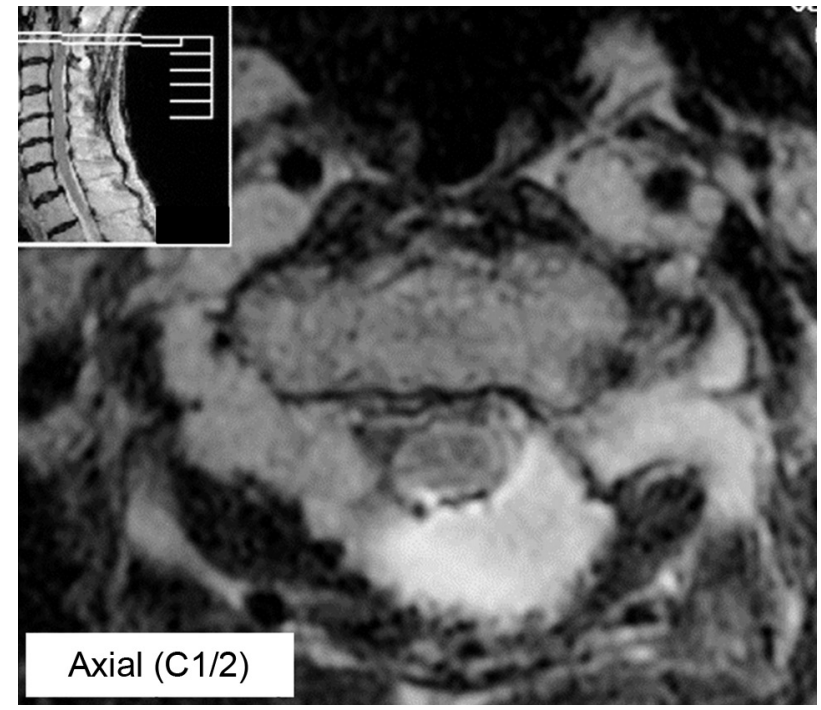

Fig. 4. Postoperative magnetic resonance images. Although bilateral foraminal portions were left, the intracanal tumor was resected completely and spinal cord decompression was sufficient. 


\section{Discussion}

This case is the fourth known example of cervical ganglioneuromas of bilateral symmetric dumbbell type. The previous three cases are as follows. Ugarriza et al. [3] reported symmetric ganglioneuromas from bilateral C2 nerve roots in a 53-year-old man. Kyoshima et al. [2] reported ganglioneuromas at bilateral C2 and C3 levels in a 53-year-old man with neurofibromatosis type 1 (NF-1). Miyakoshi et al. [1] reported multiple ganglioneuromas in a 15-year-old boy with NF-1. The present case can thus be characterized as the fourth report of this pathological condition. Importantly, the present patient is the eldest of the four cases, and in his case, while age and poor general condition were shown to be the limiting factors for surgical treatment, subtotal resection of the tumor aiming at cord decompression was successful. Therefore, the efficacy of palliative surgical treatment for symmetric dumbbell type ganglioneuroma is, we believe, worth being added to the literature.

In this case, the intradural portion of the tumor, as well as the extradural portion, was causing spinal cord compression. Therefore, resection of the intradural portion via durotomy, accompanied with debulking procedure for the extradural portions, was the chosen method. Taking into account the origin of the ganglioneuroma, the tumor arises from sympathetic nerve and there is thus the possibility of the dumbbell tumor becoming extended to the intracanal. However, in such cases, extradural extension is usual and intradural extension is very rare because the origin of these tumors is related to the sensory root ganglion and extended from the foramen outside [2]. Of the previously reported three cases, two comprised an extradural tumor and one comprised intradural extension. All cases originated at the dorsal root [1-3]. Detailed preoperative imaging diagnosis, including MRI and computed tomography myelography, would help clinicians to diagnose the precise localization of the tumor and determine surgical planning. While ganglioneuromas are most commonly found as solitary lesions, exceptionally multifocal lesions associated with von Recklinghausen's disease have also been reported [1]. In the present patient, von Recklinghausen's disease was ruled out. The patient is the oldest known case of bilateral symmetric intradural extramedullary ganglioneuroma.

Radilographically, ganglioneuromas showed a well defined, oval shape. Calcification of around 30\% was seen on computed tomography scan. MR signal intensity was mainly low on T1-weighted images and high on T2-weighted images. Pathologically, ganglioneuromas are white, firm and encapsulated, slow-growing tumors. Histologically, these tumors contain large ganglion cells and areas of smaller lymphocyte-like cells within a matrix of fibrous and Schwann cells. Additionally, multinucleate cells with a well-defined nucleolus in each nucleus are commonly found. A diagnosis of ganglioneuroma is based on the absence of necrosis or immature ganglion cells [3]. The present case fulfilled all the above criteria. Importantly, previously reported cervical symmetric dumbbell type tumors have all been ganglioneuromas [13]. As such, the combination of the above findings led us to diagnose the tumor as symmetric bilateral dumbbell shaped ganglioneuromas.

For the treatment of symptomatic solitary ganglioneuromas, there is general agreement that total resection is essential. However, for multiple spinal ganglioneuromas in which complete resection at all sites is impossible, subtotal or partial resection can be an acceptable choice [1]. In our case, several specific conditions made us hesitate to perform radical resection. First, the bilateral tumors extended outside of the foramen and reached to the vertebral artery. Total resection of tumors via posterior approach entailed the risk of vertebral artery injury. Otherwise, bilateral anterior approach would have been required to resect the bilateral extra foraminal portions. Second, the main purpose of emergency surgery was spinal cord decompression. Third, the ganglioneuromas were benign and slow growing. Fourth, the patient was aged (over 70 years) with poor systemic condition. Therefore, we performed total resection of the intracanalar tumor, aiming at complete decompression of the spinal cord, and partial and subtotal resection of the extracanalar portions.

We described an extremely rare case with bilateral and symmetric dumbbell ganglioneuromas of the cervical spine in an elderly patient. We performed total resection of the intracanalar tumor, and partial and subtotal resection of the foraminal outside portions, aiming at complete decompression of the spinal cord.

\section{Conflict of Interest}

No potential conflict of interest relevant to this article was reported. 


\section{References}

1. Miyakoshi N, Hongo M, Kasukawa Y, Misawa A, Shimada Y. Bilateral and symmetric C1-C2 dumbbell ganglioneuromas associated with neurofibromatosis type 1 causing severe spinal cord compression. Spine J 2010;10:e11-5.

2. Kyoshima K, Sakai K, Kanaji M, et al. Symmetric dumbbell ganglioneuromas of bilateral C2 and C3 roots with intradural extension associated with von Recklinghausen's disease: case report. Surg Neurol 2004;61:468-73.

3. Ugarriza LF, Cabezudo JM, Ramirez JM, Lorenzana LM, Porras LF. Bilateral and symmetric C1-C2 dumbbell ganglioneuromas producing severe spinal cord compression. Surg Neurol 2001;55:228-31.

4. Strang RR, Nordenstam H. Dumb-bell ganglioneuroma of the cervical spine. Acta Neurol Scand 1962;38:60-6.

5. Maggi G, Dorato P, Trischitta V, Varone A, Civetta
F. Cervical dumbbell ganglioneuroma in an eighteen month old child. A case report. J Neurosurg Sci 1995;39:257-60.

6. Bhand AA. Ganglioneuroma of the cervical spine. J Coll Physicians Surg Pak 2005;15:114-6.

7. Radulovi DV, Branislav D, Skender-Gazibara MK, Igor NM. Cervical dumbbell ganglioneuroma producing spinal cord compression. Neurol India 2005;53:370-1.

8. Shephard RH, Sutton D. Dumb-bell ganglioneuromata of the spine with a report of four cases. Br J Surg 1958;45:305-17.

9. Tei R, Morimoto T, Miyamoto K, et al. Intradural extramedullary ganglioneuroma associated with multiple hamartoma syndrome. Neurol Med Chir (Tokyo) 2007;47:513-5.

10. Sinclair JE, Yang YH. Ganglioneuromata of the spine associated with von Recklinghausen's disease. J Neurosurg Sci 1961;18:115-9. 